\title{
Highly Sensitive Plasmonic Refractive Index Sensor Based on Dual D-Shaped Photonic Crystal Fiber with Aluminum Nitride-Silver Films
}

\section{Sanfeng Gu}

Chongqing University

\section{Wei Sun}

China Coal Research Institute Chongqing Branch: China Coal Technology and Engineering Group Corp Chongqing Research Institute

\section{Meng Li}

Chongqing University

Ming Deng ( $\nabla$ dengming@cqu.edu.cn )

Chongqing University https://orcid.org/0000-0002-2155-987X

\section{Research Article}

Keywords: Photonic crystal fiber, Surface plasmon resonance, Finite element method, Refractive index measurement

Posted Date: December 6th, 2021

DOI: https://doi.org/10.21203/rs.3.rs-1077705/v1

License: (c) (1) This work is licensed under a Creative Commons Attribution 4.0 International License. Read Full License

Version of Record: A version of this preprint was published at Plasmonics on February 21st, 2022. See the published version at https://doi.org/10.1007/s11468-022-01609-8. 


\section{Abstract}

A dual-core and dual D-shaped photonic crystal fiber (PCF) based surface plasmon resonance (SPR) sensor with silver and Aluminum Nitride (AIN) films is designed. The distribution characteristics of the electromagnetic fields of core and plasmon modes, as well as the sensing properties are numerically studied by finite element method (FEM). The structure parameters of the designed sensor are optimized by the optical loss spectrum. The results show the resonance wavelength variation of $489 \mathrm{~nm}$ for the refractive index (RI) range of 1.36 1.42. In addition, a maximum wavelength sensitivity of $13400 \mathrm{~nm} / \mathrm{RIU}$ with the corresponding RI resolution of $7.46 \times 10^{-6} \mathrm{RIU}$ is obtained in the RI range of $1.41 \sim 1.42$. The proposed sensor with the merits of high sensitivity, low cost and simple structure has a wide application in the fields of RI sensing, such as hazardous gas detection, environmental monitoring and biochemical analysis.

\section{Introduction}

Surface plasmon resonance (SPR) as a kind of remarkable RI sensing technology shows great research value in the fields of environmental monitoring [1], medical diagnosis [2] and biochemical analysis [3] due to outstanding advantages of high sensitivity, wide operating wavelength range, free label and fast response [4-6]. Photonic crystal fiber (PCF) are well known as micro-structured fiber (MSF) with prominent virtues, such as large mode field area, low loss and endlessly single mode transmission $[7,8]$. Recently, the sensors based on PCF-SPR have achieved a great leap in the fields of fiber sensing because of flexible structure design, anti-electromag-netic interference, compactness, remotely real-time and online measurement [9-11]. A variety of PCF-SPR sensors for refractive index (RI) sensing have been reported [12-14]. Yang et al proved a PCF-SPR sensor adopting alternate holes coated with graphene-silver (G-Ag) bimetallic layers, which obtained the spectral sensitivity of up to $2520 \mathrm{~nm} / \mathrm{RIU}$ in the RI range of 1.33 1.35 [15]. Rifat et al proposed a PCF-SPR sensor with a gold layer selectively coated in a large cavity, achieving a maximum sensitivity of $11,000 \mathrm{~nm} / \mathrm{RIU}$ in the RI range of $1.33 \sim 1.42$ [16]. Chen et al designed a PCF-SPR sensor based on dual optofluidic channel, realizing a maximum wavelength sensitivity of $5500 \mathrm{~nm} / \mathrm{RIU}$ in the RI range of 1.32 1.38 [17].

The PCF-SPR sensors mentioned above have something in common, that is, the analytes or plasmonic materials are filled or deposited inside the air holes. However, it is a great challenge for filling or coating mediums into the air holes of PCF in practice. In addition, it is also very inconvenient to change the analytes RI. In order to solve the above problems, a great deal of efforts have been put into the structure design of D-shaped PCF-SPR sensors to meet different sensing and detection requirements [18-21]. For example, Wang et al confirmed a dual-polished PCF-SPR sensor covered with gold film, showing a maximum sensitivity as high as $8000 \mathrm{~nm} / \mathrm{RIU}$ in the range of 1.30 1.42 [22]. Chen et al verified a Dshaped PCF-SPR sensor with a open-ring channel coated with gold film, whose maximum sensitivity reached $11055 \mathrm{~nm} / \mathrm{RIU}$ in the range of 1.20 1.29 [23]. Tian et al reported a PCF-SPR sensor based a quasi-D-shaped layout with ITO- graphene (ITO-G) films combination, revealing a maximum sensitivity of $12,000 \mathrm{~nm} / \mathrm{RIU}$ in the range of 1.21 1.32 [24]. Fang et al demonstrated a D-shaped PCF-SPR sensor 
utilizing silver-titanium dioxide $\left(\mathrm{Ag}-\mathrm{T}_{\mathrm{i}} \mathrm{O}_{2}\right)$ composite micro-grating, obtaining a maximum sensitivity of $10,300 \mathrm{~nm} / \mathrm{RIU}$ in the range of 1.33 1.40 [25]. In addition, some sensors with tantalum pentoxide-gold

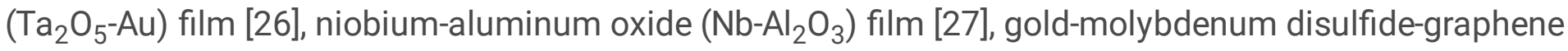
$\left(\mathrm{Au}-\mathrm{MoS}_{2}-\mathrm{G}\right)$ [28] film have also been reported. With the development of optical fiber technology and material science, some new materials integrated in the fiber sensors are used to improve the sensing performance.

In this paper, a dual D-shaped PCF-SPR sensor with the Aluminum Nitride (AIN) films coated on the silver films is designed. The PCF cladding with the D-shaped structure can be immersed directly into the liquid/gas environments and interact instantly with the analytes. In addition, the silver as the plasmonic material can form a relatively narrow spectrum to achieve the high detection accuracy. The AIN films depos-ited on the Ag film surfaces can prevent it oxidation, as well as enhance the resonance effect and detection sensitivity simultaneously. What's more, the AIN-Ag films can be easily prepared by the chemical vapor deposition technology (CVD) [29] and integrated on the surfaces of D-shaped PCF through the micro-machining technology [30]. The structure parame-ters of the designed sensor are optimized by the optical loss spectrum. The results show that the change in the resonance wavelength with $489 \mathrm{~nm}$ is obtained for the RI range of 1.36 1.42, and the maximum spectral sensitivity reaches up to $13400 \mathrm{~nm} / \mathrm{RIU}$, corresponding to the maximum RI resolu-tion of $7.46 \times 10^{-6} \mathrm{RIU}$, which has a bright application prospect in the fields of RI sensing.

\section{Sensing Model And Method}

The cross-section of the proposed PCF-SPR sensor is shown in Fig. 1(a). The cladding air holes of PCF with the radius of $r$ are arranged in a triangular lattice layout, and the space distance between the adjacent cladding air holes is $\Lambda$. Two cladding air holes distributed in the symmetric positions on the PCF center are removed to form a high RI-guided dual-core structure. Both sides of the circular fiber structure are polished into the D-shaped structures, and the distance from the D-shaped surfaces to the center of PCF is $d$. The Ag films with the thickness of $d_{2}$ as the plasmonic material are coated on the dual Dshaped PCF surfaces, and a layer of AIN film with the thickness of $d_{1}$ is deposited on the Ag film surface to protect it from oxidation and strengthen the resonance coupling between the core mode and SPR mode at the same time. The length of AlN-Ag films is the same as the space distance of the adjacent cladding air holes considering that the core mode is leaked to the AIN-Ag film surfaces through the slits between two cladding air holes. Fig. 1(b) represents the schematic diagram of the experimental devices of the propo-sed sensor. The PCF-SPR sensor is directly surrounded by the analytes with the RI of $n_{\mathrm{a}}$, which can reduce the difficulty of analytes filling and enhance the interaction between the plasmon mode and analytes. A broadband light source (BBS) as the input light is polarized by a polarization controller (PC) and then transmitted into the dual-core and dual D-shaped PCF-SPR sensor. Finally, the output light is immediately sent into the optical spectrum analyzer (OSA) to observe the corresponding loss spectrum. 
Here, the initial structure parameters are as follows: $\Lambda=4.0 \mu \mathrm{m}, r=1.2 \mu \mathrm{m}, d=8.5 \mu \mathrm{m}, d_{1}=20 \mathrm{~nm}, d_{2}=40 \mathrm{~nm}$, and $n_{\mathrm{a}}=1.40$. The above values will be used in the following numerical analysis without special instructions. In addition, the fused silica is used as the substrate material of PCF cladding, and its dispersion relationship with respect to the wavelength is described by Sellmeier equation [31], as shown in equation (1). The permittivity of Ag can be expressed by Drude model [32], as displayed in equation (2). The RI of AIN can be found in the reference [33], whose real part changes roughly from 2.14 to 2.12, and the imaginary part is zero in the wavelength range of $700-1500 \mathrm{~nm}$.

$$
\begin{gathered}
n^{2}-1=\frac{\mathrm{A}_{1} \lambda^{2}}{\lambda^{2}-\mathrm{B}_{1}^{2}}+\frac{\mathrm{A}_{2} \lambda^{2}}{\lambda^{2}-\mathrm{B}_{2}^{2}}+\frac{\mathrm{A}_{3} \lambda^{2}}{\lambda^{2}-\mathrm{B}_{3}^{2}} \\
\varepsilon_{\mathrm{Ag}}=1-\frac{\lambda^{2} \lambda_{\mathrm{c}}}{\lambda_{\mathrm{p}}^{2}\left(\lambda_{\mathrm{c}}+\mathrm{i} \lambda\right)}
\end{gathered}
$$

where $A_{1}=0.6961663, A_{2}=0.4079426, A_{3}=0.8974790, B_{1}=0.0684043 \mu \mathrm{m}, B_{2}=0.1162414 \mu \mathrm{m}$, and $\mathrm{B}_{3}=9.8961610 \mu \mathrm{m} . \lambda$ is the free-space wavelength, $\lambda_{c}=17.61400 \mu \mathrm{m}$, and $\lambda_{\mathrm{p}}=0.14541 \mu \mathrm{m}$.

According to the coupled mode theory [34], when the dispersion curves of core mode and SPR mode intersect at a certain point, in other words, when the phase matching condition is met, a strong resonance coupling between two modes will occur, resulting in a large amount of energy being transferred from the core mode to the SPR mode and producing an obvious resonance loss peak in the spectrum. As a result, we can use the confinement loss of the core mode to characterize the SPR effect, which is proportional to the imaginary part of the mode effective RI (Im(neff)) and can be defined as [35]

$$
L_{\mathrm{oss}}=40 \pi \cdot \operatorname{Im}\left(n_{\mathrm{eff}}\right) /(\ln (10) \lambda) \approx 8.68 \cdot k_{0} \cdot \operatorname{Im}\left(n_{\mathrm{eff}}\right)(\mathrm{dB} / \mathrm{cm})(3)
$$

where $k_{0}=2 \Pi / \lambda$ is the vacuum wave-number.

Firstly, we analyze the electromagnetic field characteri-stics of the designed PCF-SPR structure in the COMSOL Multiphysics software based finite element method (FEM). A perfectly matched layer (PML) is arranged outside the fiber structure in order to seek the desired modes and save computing time. Fig. 2 shows the energy distribution in the dual-core at the resonance wavelength of $1095 \mathrm{~nm}$. The red arrows is on behalf of the vector direction of the electric field. There are four core modes that can transmit stably in the dual-core, namely, $x$ and $y$-polarized odd and even modes, whose

effective RIs are 1.443429-1.752059E-5i (Fig. 2(a)), 1.443515-1.617902E-5i (Fig. 2(b)), 1.443437 (Fig. 2(c)), and 1.443506 (Fig. 2(d)), respectively. It can be seen that there is a fraction of the core mode energy leakage to the AIN-Ag film surfaces (Fig. 2(a) and (b)), on the contrary, no light field is concentrated on the AIN-Ag film surfaces (Fig. 2(c) and (d)), which illustrates that $y$-polarized odd and even modes (Fig. 2(c) and (d)) cannot excite the SPR mode. Additionally, there is a maximum value of Im(neff) for the $x$ polarized odd mode (Fig. 2(a)), which indicates that the coupling strength between the $x$-polarized odd 
mode (Fig. 2(a)) and SPR mode is higher than that of $x$-polarized even mode (Fig. 2(b)). As a consequence, the $x$-polarized odd mode (Fig. 2(a)) is used to assess the sensing performance in the following research.

In order to understand the energy transfer process better, the phase matching relationship between the core mode and SPR mode is plotted in Fig. 3. It can be noticed that the real parts of the effective RI $(\operatorname{Re}($ neff)) of core mode and SPR mode both reduce with the increment of incident wavelength. When the $\operatorname{Re}$ (neff) of two modes is equal at point $(m)$, the phase matching condition is satisfied, causing more energy of the core mode leakage from dual-core to AIN-Ag film surfaces, which leads to a distinct loss peak at the resonance wavelength of $1095 \mathrm{~nm}$ in the spectrum. In addition, the phase matching relationship will be satisfied renewedly with the analyte RI changing, resulting in the resonance wavelength shift. Therefore, the corresponding relationship of the resonance wavelength in relation to the analyte RI can be established to achieve the RI sensing. Inset (a) shows the electric field distribution of $x$ polarized core mode, and the light field is almost entirely gathered in the dual-core at the non-resonance wavelengths. Inset (b) is the optical field distribution of SPR mode, whose light field is concentrated on the AIN-Ag film surfaces. The energy distribution at the resonance wavelength (point $(\mathrm{m})$ ) is drawn in Inset (c), and the obvious energy transfer from the dual-core to AIN-Ag film surfaces is observed, which generates the strong resonance coupling between the core mode and SPR mode.

\section{Results And Discussions}

\section{Structure parameters optimization}

The AIN films as the dielectric layers have the strong depend-ence on the designed sensor performance. Fig. 4 shows the loss spectrum of the core mode for different AIN thickness $\left(d_{1}\right)$. The calculated resonance wavelengths are 916,996 and $1092 \mathrm{~nm}$ for $n_{\mathrm{a}}$ of 1.39, as well as 990,1095 and $1170 \mathrm{~nm}$ for $n_{\mathrm{a}}$ of 1.40 due to $d_{1}$ of 15,20 and $25 \mathrm{~nm}$, respectively. In addition, when $n_{\mathrm{a}}$ varies from 1.39 to 1.40 , the changes in the resonance wavelength are 74,99 and $78 \mathrm{~nm}$, corresponding to the wavelength sensitivities of 7400,9900 and $7800 \mathrm{~nm} / \mathrm{RIU}$. There is a maximum spectral sensitivity of $9900 \mathrm{~nm} / \mathrm{RIU}$ for $d_{1}=20 \mathrm{~nm}$. So $d_{1}=20 \mathrm{~nm}$ is chosen.

The Ag films as the region where the plasmon mode is excited have an important impact on the resonance peak intensity and position. Fig. 5 displays the effect of different Ag films thickness (d2) on the loss spectrum. When $n_{\mathrm{a}}$ is 1.39 and 1.40 , the resonance wavelengths both shift towards long wavelengths as $d_{1}$ increases from 35 to $45 \mathrm{~nm}$ with a step of $5 \mathrm{~nm}$. Specifically, the resonance wavelengths are 958,996 , and $1039 \mathrm{~nm}$ for $n_{\mathrm{a}}$ of 1.39 , as well as 1027,1095 and $1125 \mathrm{~nm}$ for $n_{\mathrm{a}}$ of 1.40 , corresponding to $d_{2}$ of 35,40 and $45 \mathrm{~nm}$, respectively. Additionally, the variations of the resonance wavelengths are 69, 99 and $86 \mathrm{~nm}$, corresponding to the wavelength sensitivities of 6900,9900 and 8600 $\mathrm{nm} /$ RIU. The $d_{2}=40 \mathrm{~nm}$ selected as the optimal value realizes a maxi-mum wavelength sensitivity of 9900 $\mathrm{nm} / \mathrm{RIU}$. 
The confinement loss of the core mode is caused by the finiteness of the cladding air holes. As a result, the positions of the loss peak have strong dependence on the radius of the cladding air holes ( $r$ ). Fig. 6(a) represents the loss spectrum of the core mode for different $r$. The results show that the resonance intensity diminishes from 20.567062 to $3.364749 \mathrm{~dB} / \mathrm{cm}$ with the $r$ increasing from 1.1 to $1.3 \mu \mathrm{m}$ with a step of $0.05 \mu \mathrm{m}$. It can be explained by the fact that the greater the cladding air holes are, the smaller the equivalent RI of the cladding is, resulting in the Re(neff) difference between the dual-core and PCF cladding becoming large gradually, which leads to more energy being confined to the dual-core. In addition, the resonance wavelengths are almost unchanged and the full width at a half maximum (FWHM) of the loss spectrum expands with the increment of $r$. When $n_{\mathrm{a}}$ is 1.39 and 1.40 , the effect of different $r$ on the changes of the resonance wavelength is described in Fig. $6(\mathrm{~b})$. The varia-tions of the resonance wavelength enlarge from 91 to $112 \mathrm{~nm}$, while the resonance strength reduces as the $r$ increases in the calculated ranges. As a consequence, $r=1.2 \mu \mathrm{m}$ is selected considering a compromise between the wavelength sensiti-vity and the resonance depth.

The spectral properties can be affected by the distance $(d)$ from the D-shaped surfaces to the center of PCF, as shown in Fig. 7. In general, when na is 1.39 or 1.40 , the resonance wavelengths create the redshift and the resonance intensity damps as $d$ increases from 8.3 to $8.7 \mu \mathrm{m}$ with a step of $0.2 \mu \mathrm{m}$. The shifts of resonance wavelength induced by the $n_{\mathrm{a}}$ changing between 1.39 and 1.40 are 118,99 and $92 \mathrm{~nm}$ for $\mathrm{d}$ of $8.3,8.5$ and $8.7 \mu \mathrm{m}$, corresponding to the wavelength sensitivities of 11800,9900 and $9200 \mathrm{~nm} / \mathrm{RIU}$, respectively. In addition, it can be found that there are two small loss peaks when $d$ is $8.3 \mu \mathrm{m}$, whose positions are severally located at the resonance wavelength of $1043 \mathrm{~nm}$ for $n_{\mathrm{a}}$ of 1.39 , and $1022 \mathrm{~nm}$ for $n_{\mathrm{a}}$ of 1.40 . It may be responsible for the resonance coupling between the core mode and SPR mode with higher order, which makes the spectrum untidy and is adverse for the RI sensing and measurement. Therefore, $d=8.5 \mu \mathrm{m}$ as an optimized value is adopted in the following analysis.

The spectral properties can be affected by the distance $(d)$ from the D-shaped surfaces to the center of PCF, as shown in Fig. 7. In general, when $n_{\mathrm{a}}$ is 1.39 or 1.40 , the resonance wavelengths create the redshift and the resonance intensity damps as $d$ increases from 8.3 to $8.7 \mu \mathrm{m}$ with a step of $0.2 \mu \mathrm{m}$. The shifts of resonance wavelength induced by the $n_{\mathrm{a}}$ changing between 1.39 and 1.40 are 118,99 and $92 \mathrm{~nm}$ for $d$ of $8.3,8.5$ and $8.7 \mu \mathrm{m}$, corresponding to the wavelength sensitivities of 11800,9900 and $9200 \mathrm{~nm} / \mathrm{RIU}$, respectively. In addition, it can be found that there are two small loss peaks when $d$ is $8.3 \mu \mathrm{m}$, whose positions are severally located at the resonance wavelength of $1043 \mathrm{~nm}$ for $n_{\mathrm{a}}$ of 1.39 , and $1022 \mathrm{~nm}$ for $n_{\mathrm{a}}$ of 1.40 . It may be responsible for the resonance coupling between the core mode and SPR mode with higher order, which makes the spectrum untidy and is adverse for the RI sensing and measurement. Therefore, $d=8.5 \mu \mathrm{m}$ as an optimized value is adopted in the following analysis.

\section{Performance analysis}

Generally, the sensitivity of the designed PCF-SPR sensor can be characterized by two ways, namely, wavelength and intensity interrogation modes. Here, the sensor performance is evaluated in a wavelength demodulation mode [36], whose sensitivity can be expressed as equation (4). At the same time, the 
detection resolution as another important index is used to assess the minimum RI change that causes the spectral response [37], as shown in equation (5).

$$
\begin{aligned}
& S(\mathrm{~nm} / \mathrm{RIU})=\Delta \lambda_{\text {peak }} / \Delta n_{\mathrm{a}} \\
& R(\mathrm{RIU})=\Delta \eta_{\mathrm{a}} \times \Delta \lambda_{\min } / \Delta \lambda_{\text {peask }}
\end{aligned}
$$

where $\Delta \lambda_{\text {peak }}$ denotes the resonance wavelength variation as a response to the change $\left(\Delta n_{\mathrm{a}}\right)$ in the analyte $\mathrm{Rl}$, and $\Delta \lambda_{\min }$ is set to $0.1 \mathrm{~nm}$ as the wavelength resolution of the spectral analyzer.

The detection performance of the designed PCF-SPR sensor is evaluated for the analyte RI $\left(n_{\mathrm{a}}\right)$ varying from 1.36 to 1.42 with a step of 0.01. Fig. 8(a) shows the loss spectrum of the core mode for different $n_{\mathrm{a}}$. The resonance depth enlarges from 2.737063 to $99.575642 \mathrm{~dB} / \mathrm{cm}$ with $n_{\mathrm{a}}$ increas-ing in the calculated range. Meanwhile, the resonance wave-length shifts towards long wavelength from 839 to $1328 \mathrm{~nm}$, whose changes in the resonance wavelength are $489 \mathrm{~nm}$, corresponding to an average wavelength sensitivity of $8150 \mathrm{~nm} /$ RIU. Fig. 8(b) represents the fitting relationship between

the resonance wavelength and $n_{\mathrm{a}}$. The polynomial fitting function with the second order is adopted to describe the relationship between the resonance wavelength and $n_{a}$, as shown in equation (6), which obtains a better fitting precision of R-square $=0.9992$. In addition, it can easily acquire the sensitivity near a certain $n_{\mathrm{a}}$ through taking the derivative of the resonance wavelength in relation to $n_{\mathrm{a}}$ according to the equation (6).

$$
y=\left(a x^{2}-b x+c\right) \times 10^{5}
$$

where $a=0.9060, b=2.4382, c=1.6488$.

In order to further characterize the designed sensor performance, the wavelength sensitivity and RI resolution for different $\mathrm{RI}$ sensing regions are presented in Table 1. It can be seen that the wavelength sensitivities are 4400, 5200,6100, 9900, 9900 and $13400 \mathrm{~nm} / \mathrm{RIU}$ due to different RI range of 1.36 1.37, 1.37 1.38, 1.38 1.39, 1.39 1.40, 1.40 1.41, and 1.41 1.42, corresponding to the RI resolution improving from $2.27 \times 10^{-5}$ to $7.46 \times 10^{-6} \mathrm{RIU}$. A maximum wavelength sensitivity of $13400 \mathrm{~nm} / \mathrm{RIU}$ with the $\mathrm{RI}$ resolution of $7.46 \times 10^{-6} \mathrm{RIU}$ is obtained. In addition, we make a perform-ance comparison between the designed sensor and the report-ed PCF-SPR sensors in Table 2. It is no doubt that our sensor can achieve better sensing performance (maximum sensiti-vity and resolution) than that of the PCF-SPR sensors listed in Table 2 except Ref. 28. 
Table 1

Performance analysis of the proposed PCF-SPR sensor

\begin{tabular}{|lllll|}
\hline $\begin{array}{l}\text { Refractive index } \\
(\mathrm{RIU})\end{array}$ & $\begin{array}{l}\text { Wavelength } \\
(\mathrm{nm})\end{array}$ & $\begin{array}{l}\text { Sensitivity } \\
(\mathrm{nm} / \mathrm{RIU})\end{array}$ & $\begin{array}{l}\text { Resolution } \\
(\mathrm{RIU})\end{array}$ & $\begin{array}{l}\text { Depth } \\
(\mathrm{dB} / \mathrm{cm})\end{array}$ \\
\hline 1.36 & 839 & 4400 & $2.27 \times 10^{-5}$ & 2.737063 \\
\hline 1.37 & 883 & 5200 & $1.92 \times 10^{-5}$ & 3.621325 \\
\hline 1.38 & 935 & 6100 & $1.64 \times 10^{-5}$ & 4.968688 \\
\hline 1.39 & 996 & 9900 & $1.01 \times 10^{-5}$ & 6.695344 \\
\hline 1.40 & 1095 & 9900 & $1.01 \times 10^{-5}$ & 8.721958 \\
\hline 1.41 & 1194 & 13400 & $7.46 \times 10^{-6}$ & 28.289090 \\
\hline 1.42 & 1328 & N/A & N/A & 99.575642 \\
\hline
\end{tabular}


Table 2

Performance comparison between the designed sensor and the existing PCF-SPR sensors

\begin{tabular}{|c|c|c|c|c|c|}
\hline Ref & Sensor type & $\begin{array}{l}\text { Refractive } \\
\text { index } \\
\text { (RIU) }\end{array}$ & $\begin{array}{l}\text { Maximum } \\
\text { sensitivity } \\
\text { (nm/RIU) }\end{array}$ & $\begin{array}{l}\text { Maximum } \\
\text { resolution } \\
\text { (RIU) }\end{array}$ & Year \\
\hline [18] & D-shaped sensor with Au film & $1.33 \sim 1.38$ & 10493 & $9.53 \times 10^{-6}$ & 2017 \\
\hline [19] & D-shaped sensor with Au belts & $1.15 \sim 1.36$ & 12600 & $7.94 \times 10^{-6}$ & 2020 \\
\hline [20] & 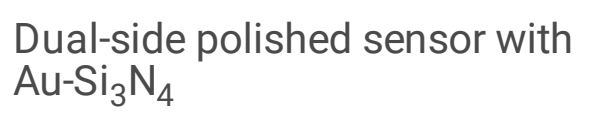 & $1.395 \sim 1.415$ & 12400 & $8.06 \times 10^{-6}$ & 2019 \\
\hline [21] & $\begin{array}{l}\text { Quasi D-shaped sensor with G- } \\
\text { ITO film }\end{array}$ & $1.33 \sim 1.38$ & 10693 & $9.35 \times 10^{-6}$ & 2017 \\
\hline [22] & $\begin{array}{l}\text { Dual-side polished sensor with } \\
\text { Au films }\end{array}$ & $1.30 \sim 1.42$ & 8000 & $1.30 \times 10^{-5}$ & 2019 \\
\hline [23] & $\begin{array}{l}\text { D-shaped sensor with Au film } \\
\text { opening }\end{array}$ & $1.20 \sim 1.29$ & 11055 & $9.05 \times 10^{-6}$ & 2018 \\
\hline [24] & $\begin{array}{l}\text { D-shaped sensor with G-ITO } \\
\text { film }\end{array}$ & $1.21 \sim 1.32$ & 12000 & $8.33 \times 10^{-6}$ & 2021 \\
\hline [25] & $\begin{array}{l}\text { D-shaped sensor with } \\
\text { segmented } \mathrm{TiO}_{2} \text {-Ag films }\end{array}$ & $1.33 \sim 1.40$ & 10300 & $9.71 \times 10^{-6}$ & 2021 \\
\hline [27] & Sensor with $\mathrm{Al}_{2} \mathrm{O}_{3}-\mathrm{Nb}$ film & $1.36 \sim 1.41$ & 8000 & $1.25 \times 10^{-5}$ & 2018 \\
\hline [28] & $\begin{array}{l}\text { D-shaped sensor with G-MoS }{ }_{2}^{-} \\
\mathrm{Au}\end{array}$ & $1.33 \sim 1.40$ & 14933.34 & $6.69 \times 10^{-6}$ & 2020 \\
\hline [38] & Sensor with TiN-Ag fim & $1.32 \sim 1.34$ & 7000 & - & 2019 \\
\hline Proposed & Sensor with AIN-Ag films & $1.36 \sim 1.42$ & 13400 & $7.46 \times 10^{-6}$ & - \\
\hline
\end{tabular}

\section{Conclusion}

A PCF-SPR sensor based dual-core and dual D-shaped structures with the AIN-Ag composite film for RI measure-ment is numerically analyzed by FEM. The structure design of D-shaped PCF strengthens the interaction between the evanescent field and analytes, as well as overcomes the packaging difficulty of liquid filling. Firstly, the electromag-netic coupling characteristics between the core mode and SPR mode are studied. Then, the structure parameters of the designed sensor are optimized by utilizing the optical loss spectrum, which shows the resonance wavelength shift of $489 \mathrm{~nm}$ in the RI range of 1.36 1.42, and a maximum wave-length sensitivity of $13400 \mathrm{~nm} / \mathrm{RIU}$ with the corresponding RI resolution of $7.46 \times 10^{-6}$ RIU. Finally, we make a perform-ance comparison between the proposed sensor and the report-ed PCF- 
SPR sensors, which indicates that the introduction of AIN film makes great contribution to the improvement of detection sensitivity. As a consequence, the designed sensor realizes better detection performance, and has a potential prospect in the fields of RI sensing.

\section{Declarations}

Funding This work was supported by the National Natural Science Foundation of China (62075022); Chongqing Technology Innovation and Development Project (cstc2020jscx-msxmX0216); Chongqing National Science Foundation of Innovative Reach Groups (cstc2020jcyj-cxttX0005); Special Project of Science and Technology Innovation and Entrepreneurship Fund of Tiandi Technology Co., Ltd (2020-TDZD007); Science and Technology Plan Project Support of Jiulongpo District (2020-02-004-Z).

Author Contribution All authors contributed to the study conception and design. Data collection was performed by Sanfeng Gu and Meng Li. Data analysis was performed by Sanfeng Gu, Wei Sun and Ming Deng. The first draft of the manuscript was written by Sanfeng Gu. Data curation were performed by Sanfeng Gu and Ming Deng. Visualization was performed by Meng Li. Supervision was performed by Ming Deng. Funding acquisition was performed by Wei Sun and Ming Deng. All authors have read and agreed to the published version of the manuscript.

Availability of Data and Materials All data, models, and code generated or used during the study appear in the submitted manuscript

Ethical Approval All authors declare that this manuscript is original and has not been published elsewhere nor is it currently under consideration for publication elsewhere. We understand that the corresponding author is the sole contact for the editorial process.

Consent to Participate We confirm that the manuscript has been read and approved by all named authors and that there are no other persons who satisfied the criteria for authorship but are not listed. We further confirm that the order of authors listed in the manuscript has been approved by all of us. All authors read and approved the final manuscript.

Consent for Publication We would like to draw the attention of the editor to the following publications of one or more of us that refer to aspects of the manuscript presently being submitted where relevant copies of such publications are attached. We further confirm that the order of authors listed in the manuscript has been approved by all of us.

Competing Interests The authors declare no competing interests.

\section{References}

1. Kim YC, Cramer J, Battaglia T, Jordan JA, Banerji SN, Peng W, Kegel LL, Booksh KS (2013) Investigation of in situ surface plasmon resonance spectroscopy for environmental monitoring in 
and around deep-sea hydrothermal vents. Anal Lett 46(10): 1607-1617.

2. Goodrich TT, Lee HJ, Corn RM (2004) Direct detection of genomic DNA by enzymatically amplified SPR imaging measurements of RNA microarrays. J Am Chem Soc 126(13): 4086-4087.

3. Liedberg B, Nylander C, Lundström I (1995) Biosensing with surface plasmon resonance-how it all started. Biosens Bioelectron 10(8): R1-R9.

4. Liu Y, Peng W (2021) Fiber-optic surface plasmon resonance sensors and biochemical applications: A review. J Lightwave Technol 39(12): 3781-3791.

5. Islam MS, Islam MR, Sultana J, Abbott D (2019) Exposed-core localized surface plasmon resonance biosensor. J Opt Soc Am B 36(8): 2306-2311.

6. Chen Q, Liang L, Zheng QL, Zhang YX, Wen L (2020) On-chip readout plasmonic mid-IR gas sensor. Opto-Electron Adv 3(7): 190040.

7. Tefelska MM, Ertman S, Wolinski TR, Mergo P, Dabrowski R (2012) Large area multimode photonic band-gap propagation in photonic liquid-crystal fiber. IEEE Photonic Tech L 24(8): 631-633.

8. Birks TA, Knight JC, Russell PSJ (1997) Endlessly singlemode photonic crystal fiber. Opt Lett 22(13): 961-963.

9. Hassani A, Skorobogatiy M (2006) Design of the microstructured optical fiber-based surface plasmon resonance sensors with enhanced microfluidics. Opt Express 14(24): 11616-11621.

10. Wu JJ, Li SG, Wang XY, Shi M, Feng XX, Liu YD (2018) Ultrahigh sensitivity refractive index sensor of a D-shaped PCF based on surface plasmon resonance. Appl Optics 57(15): 4002-4007.

11. Haider F, Mashrafi M, Haider R, Aoni RA, Ahmed R (2020) Asymmetric core-guided polarizationdependent plasmonic biosensor. Appl Optics 59(26): 7829-7835.

12. Fan ZK, Li SG, Liu Q, An GW, Chen HL, Li JS, Chao D, Li H, Zi JC, Tian WL (2015) High sensitivity of refractive index sensor based on analyte-filled photonic crystal fiber with surface plasmon resonance. IEEE Photonics J 7(3): 4800809.

13. Yan X, Fu R, Cheng TL, Li SG (2021) A highly sensitive refractive index sensor based on a V-shaped photonic crystal fiber with a high refractive index range. Sensors 21(11): 3782.

14. Islam MR, Iftekher N, Hasan R, Nayen J, Islam S (2020) Dual-polarized highly sensitive surfaceplasmon-resonance-based chemical and biomolecular sensor. Appl Optics 59(11): 3296-3305.

15. Yang XC, Lu Y, Liu BL, Yao JQ (2017) Analysis of graphene-based photonic crystal fiber sensor using birefringence and surface plasmon resonance. Plasmonics 12(2): 489-496.

16. Rifat AA, Haider F, Ahmed R, Mahdiraji GA, Adikan FRM, Miroshnichenko AE (2018) Highly sensitive selectively coated photonic crystal fiber-based plasmonic sensor. Opt Lett 43(4): 891-894.

17. Chen N, Chang M, Lu XL, Zhou J, Zhang XD (2019) Photonic crystal fiber plasmonic sensor based on dual optofluidic channel. Sensors 19(23): 5150.

18. An GW, Hao XP, Li SG, Yan X, Zhang XN (2017) D-shaped photonic crystal fiber refractive index sensor based on surface plasmon resonance. Appl Optics 56(24): 6988-6992. 
19. Zeng WY, Wang QL, Xu L (2020) Plasmonic refractive index sensor based on D-shaped photonic crystal fiber for wider range of refractive index detection. Optik 223: 165463.

20. Chen N, Chang M, Zhang XD, Zhou J, Lu XL, Zhuang SL (2019) Highly sensitive plasmonic sensor based on a dual-side polished photonic crystal fiber for component content sensing applications. Nanomaterials 9(11): 1587.

21. An GW, Li SG, Wang HY, Zhang XN (2017) Metal oxide-graphene-based quasi-D-shaped optical fiber plasmonic biosensor. IEEE Photonics J 9(4): 1-9.

22. Wang S, Li SG (2019) Surface plasmon resonance sensor based on symmetrical side-polished dualcore photonic crystal fiber. Opt Fiber Technol 51: 96-100.

23. Chen X, Xia L, Li C (2018) Surface plasmon resonance sensor based on a novel D-shaped photonic crystal fiber for low refractive index detection. IEEE Photonics J 10(1): 6800709.

24. Tian JG, Xu C, Cui SY, Ma L, Fu YQ (2021) A photonic crystal fiber-based biosensor with quasi-Dshaped layout and ITO-graphene combination. Plasmonics 16(5): 1451-1460.

25. Fang HR, Wei CJ, Yang HR, Zhao B, Yuan L, Li J (2021) D-shaped photonic crystal fiber plasmonic sensor based on silver-titanium dioxide composite micro-grating. Plasmonics 16(6): 2049-2059.

26. Li DM, Zhang W, Liu H, Hu JF, Zhou GY (2017) High sensitivity refractive index sensor based on multicoating photonic crystal fiber with surface plasmon resonance at near-infrared wavelength. IEEE Photonics J 9(2): 6801608.

27. Hasan MR, Akter S, Ahmed K, Abbott D (2018) Plasmonic refractive index sensor employing niobium nanofilm on photonic crystal fiber. IEEE Photonic Tech L 30(4): 315-318.

28. Singh S, Prajapati YK (2019) Dual-polarized ultrahigh sensitive gold/MoS2/graphene based Dshaped PCF refractive index sensor in visible to near-IR region. Opt Quant Electron 52(1): 17.

29. Sazio PJA, Amezcua-Correa A, Finlayson CE, Hayes JR, Scheidemantel TJ, Baril NF, Jackson BR, Won DJ, Zhang F, Margine ER, Gopalan V, Crespi VH, Badding JV (2006) Microstructured optical fibers as high-pressure microfluidic reactors. Science 311(5767): 1583-1586.

30. Solgaard O, Godil AA, Howe RT, Lee LP, Peter YA, Zappe H (2014) Optical MEMS: from micromirrors to complex systems. J Microelectromech S 23(3): 517-538.

31. Wang FM, Sun ZJ, Liu C, Sun T, Chu PK (2017) A highly sensitive dual-core photonic crystal fiber based on a surface plasmon resonance biosensor with silver-graphene layer. Plasmonics 12(6): 1847-1853.

32. Jing JY, Wang Q, Wang BT (2018) Refractive index sensing characteristics of carbon nanotubedeposited photonic crystal fiber SPR sensor. Opt Fiber Technol 43: 137-144.

33. Kischkat J, Peters S, Gruska B, Semtsiv M, Chashnikova M, Klinkmüller M, Fedosenko O, Machulik S, Aleksandrova A, Monastyrskyi G, Flores Y, Masselink WT (2012) Mid-infrared optical properties of thin films of aluminum oxide, titanium dioxide, silicon dioxide, aluminum nitride, and silicon nitride. Appl Optics 51(28): 6789-6798. 
34. Shuai BB, Xia L, Zhang YT, Liu DM (2012) A multi-core holey fiber based plasmonic sensor with large detection range and high linearity. Opt Express 20(6): 5974-5986

35. Otupiri R, Akowuah EK, Haxha S (2015) Multi-channel SPR biosensor based on PCF for multi-analyte sensing applications. Opt Express 23(12): 15716-15727.

36. Liu C, Su WQ, Liu Q, Lu XL, Wang FM, Sun T, Chu PK (2018) Symmetrical dual D-shape photonic crystal fibers for surface plasmon resonance sensing. Opt Express 26(7): 9039-9049.

37. Hautakorpi M, Mattinen M, Ludvigsen H (2008) Surface plasmon resonance sensor based on threehole microstructured optical fiber. Opt Express 16(12): 8427-8432.

38. El-Saeed AH, Khalil AE, Hameed MFO, Azab MY, Obayya SSA (2019) Highly sensitive SPR PCF biosensors based on Ag/TiN and Ag/ZrN configurations. Opt Quant Electron 51(2): 56-73.

\section{Figures}




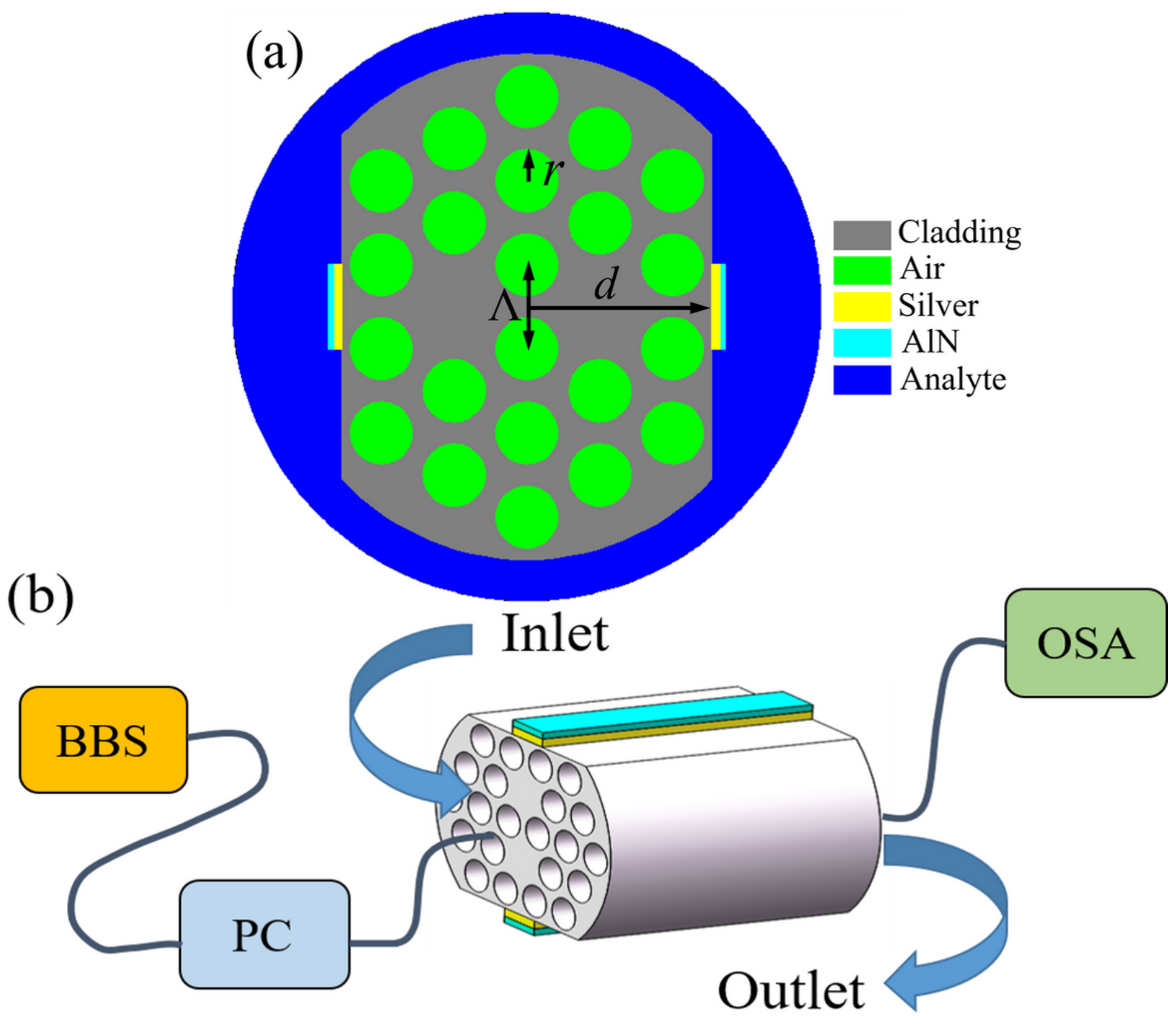

Figure 1

Diagrams of (a) cross-section and (b) experimental set-up of the designed sensor 
Effective mode index=1.443429-1.752059E-5i Surface: Electric field norm (V/m) Arrow Line: Electric field

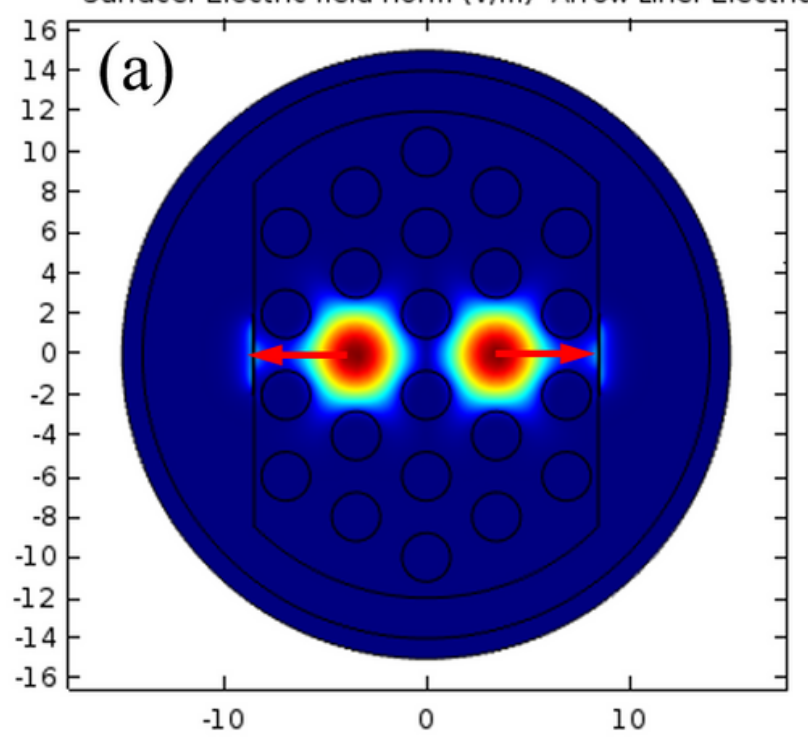

Effective mode index $=1.443437$ Surface: Electric field norm (V/m) Arrow Line: Electric field

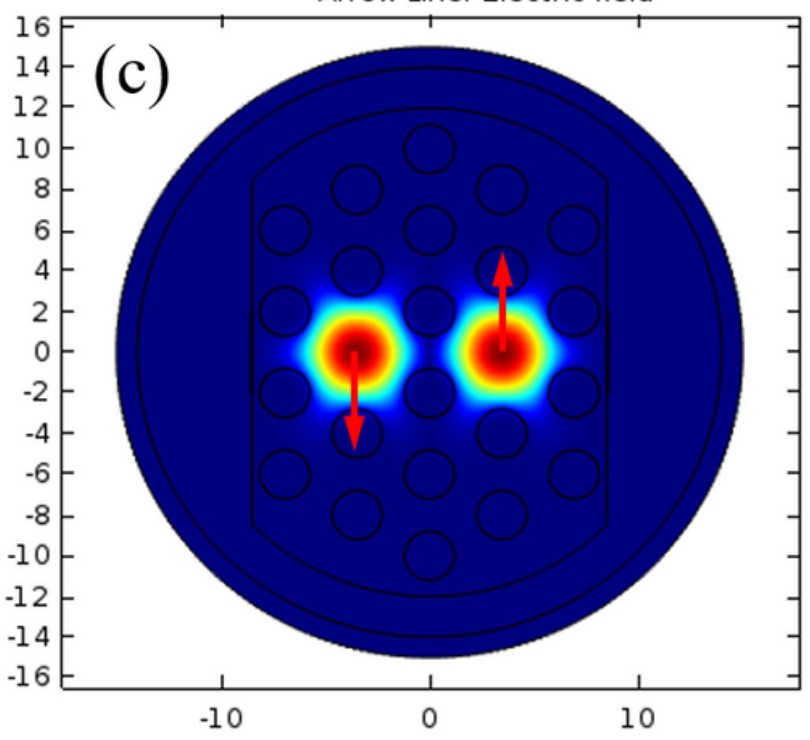

Effective mode index=1.443515-1.617902E-5i Surface: Electric field norm $(\mathrm{V} / \mathrm{m})$ Arrow Line: Electric field

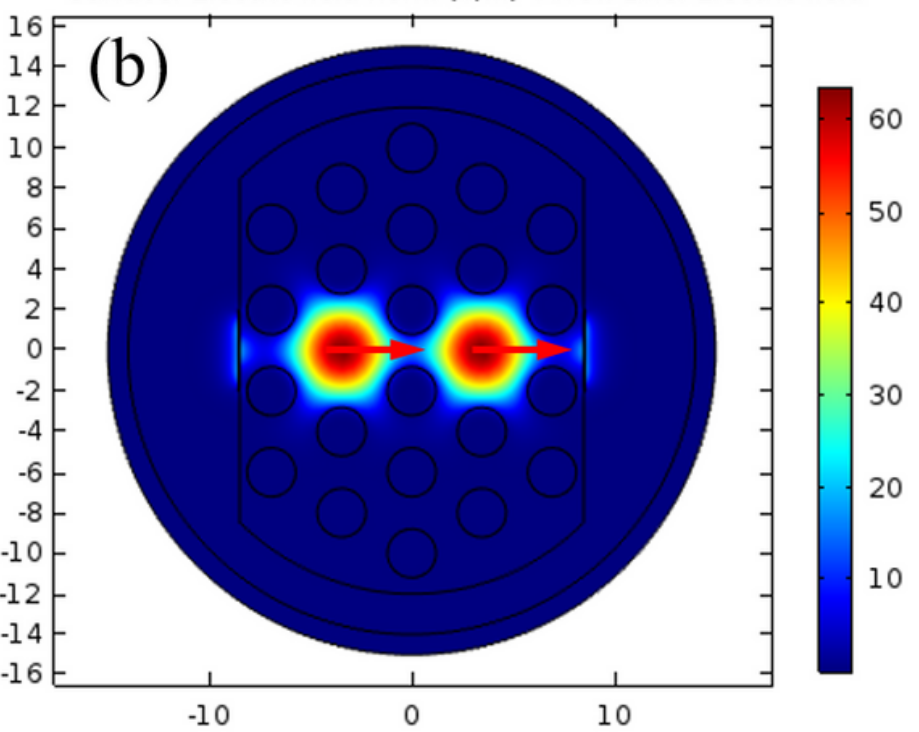

Effective mode index $=1.443506$ Surface: Electric field norm $(\mathrm{V} / \mathrm{m})$ Arrow Line: Electric field

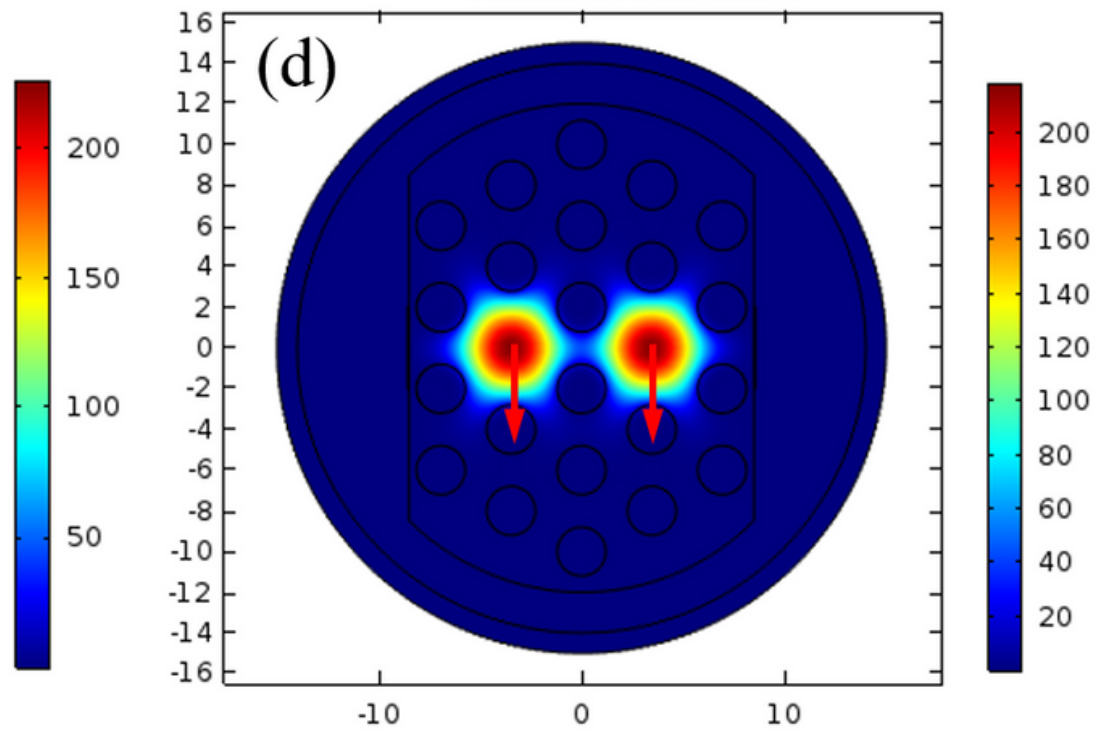

Figure 2

Electric field distribution of the core modes. (a) x-polarized odd mode and (b) even mode, (c) y-polarized odd mode and (d) even mode 


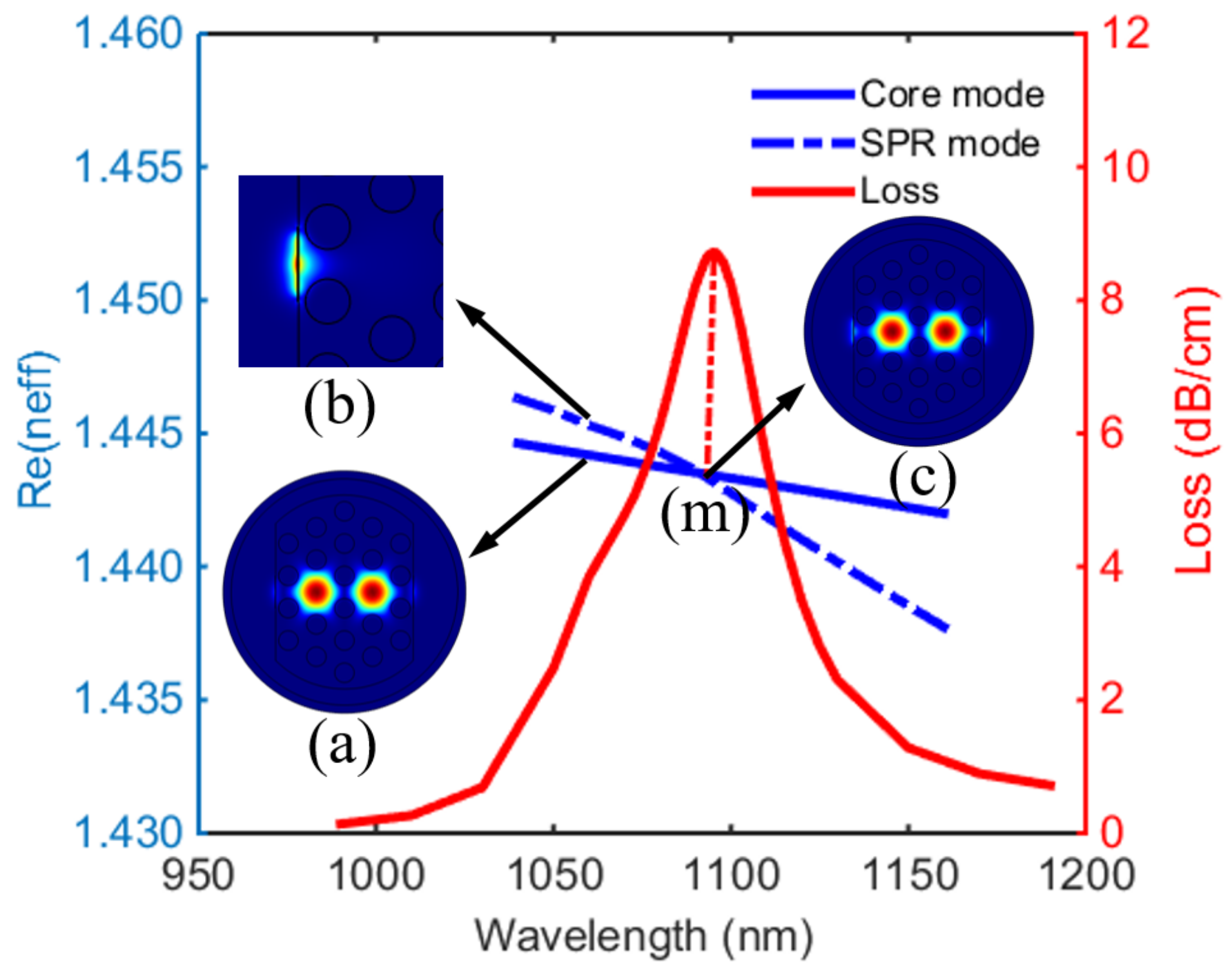

Figure 3

Dispersion relationship between the core mode and SPR mode, and loss spectrum with insets (a), (b) and (c) showing the mode field distribution 


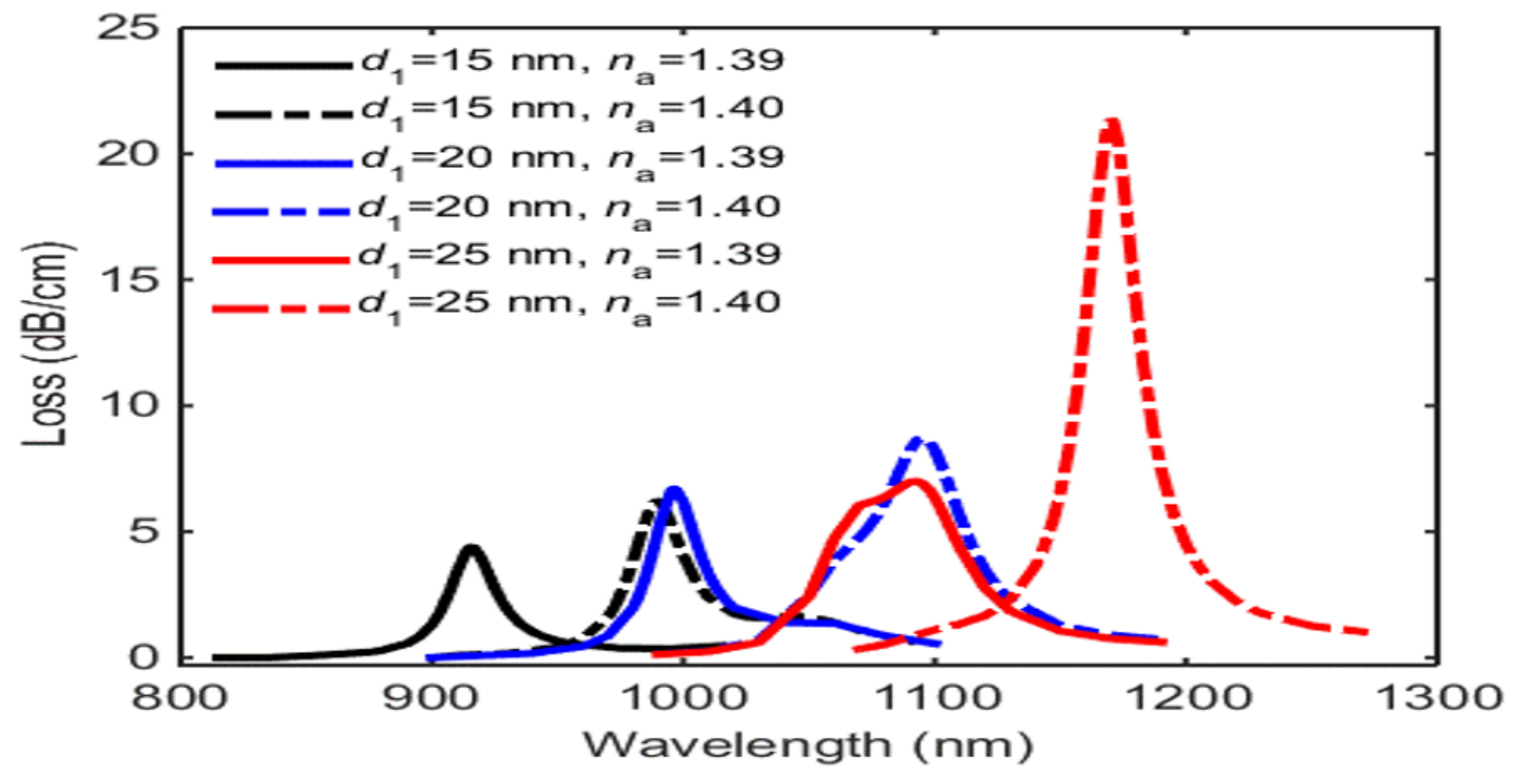

Figure 4

Loss spectrum of the core mode for different d 1

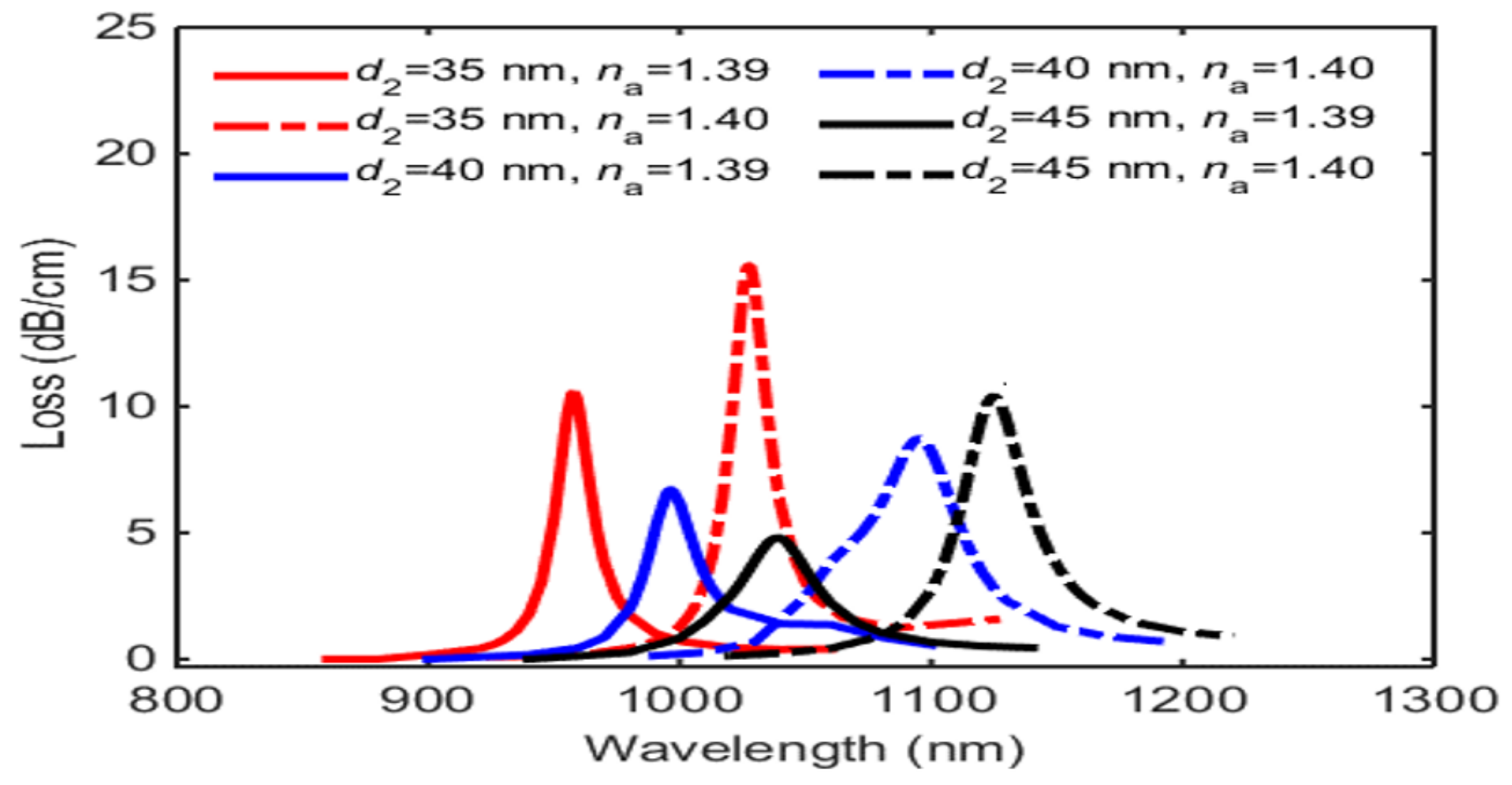

Figure 5

Loss spectrum of the core mode for different $d 2$ 

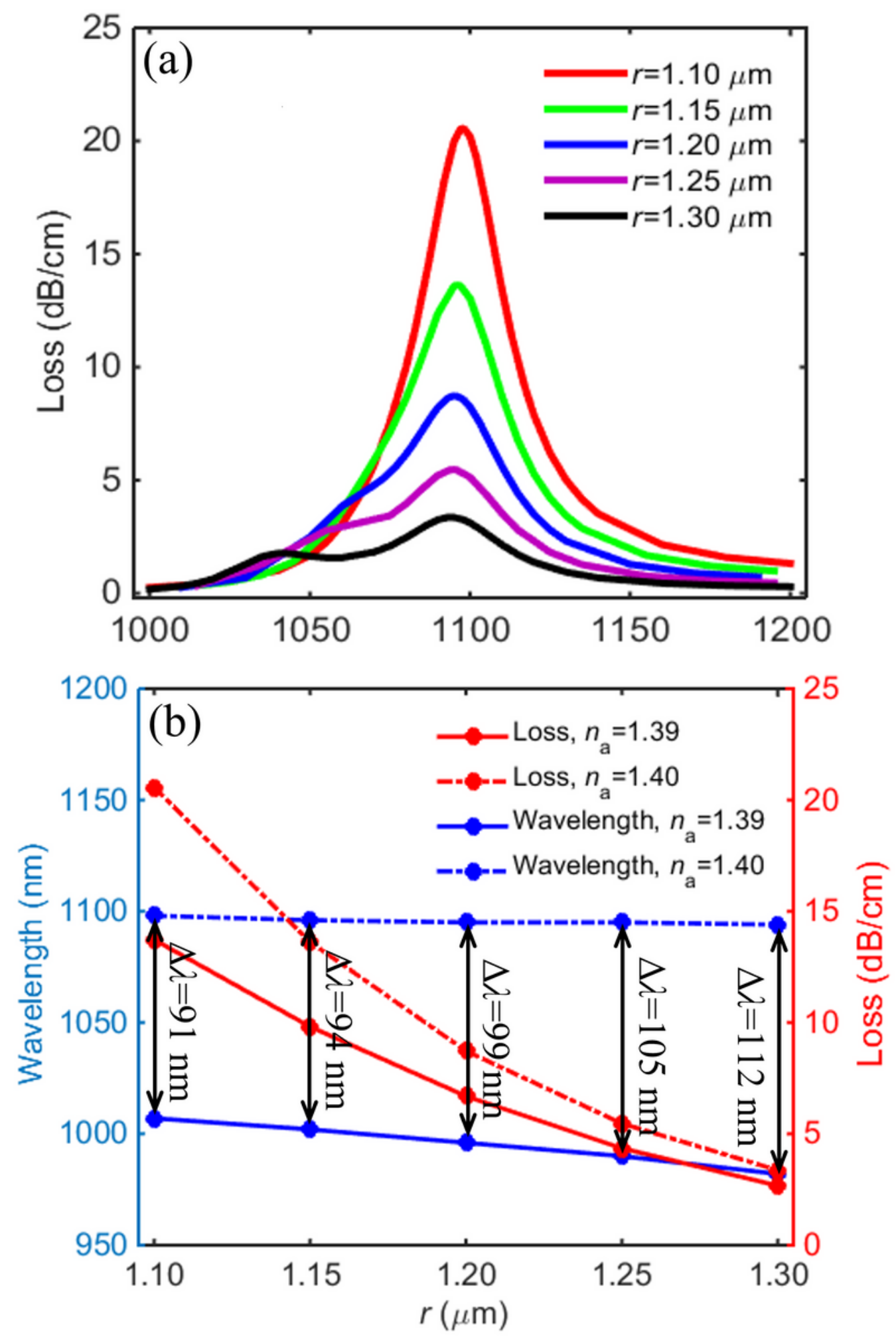

Figure 6

(a) Loss spectrum of the core mode, (b) Resonance wavelength and loss for different $r$ 


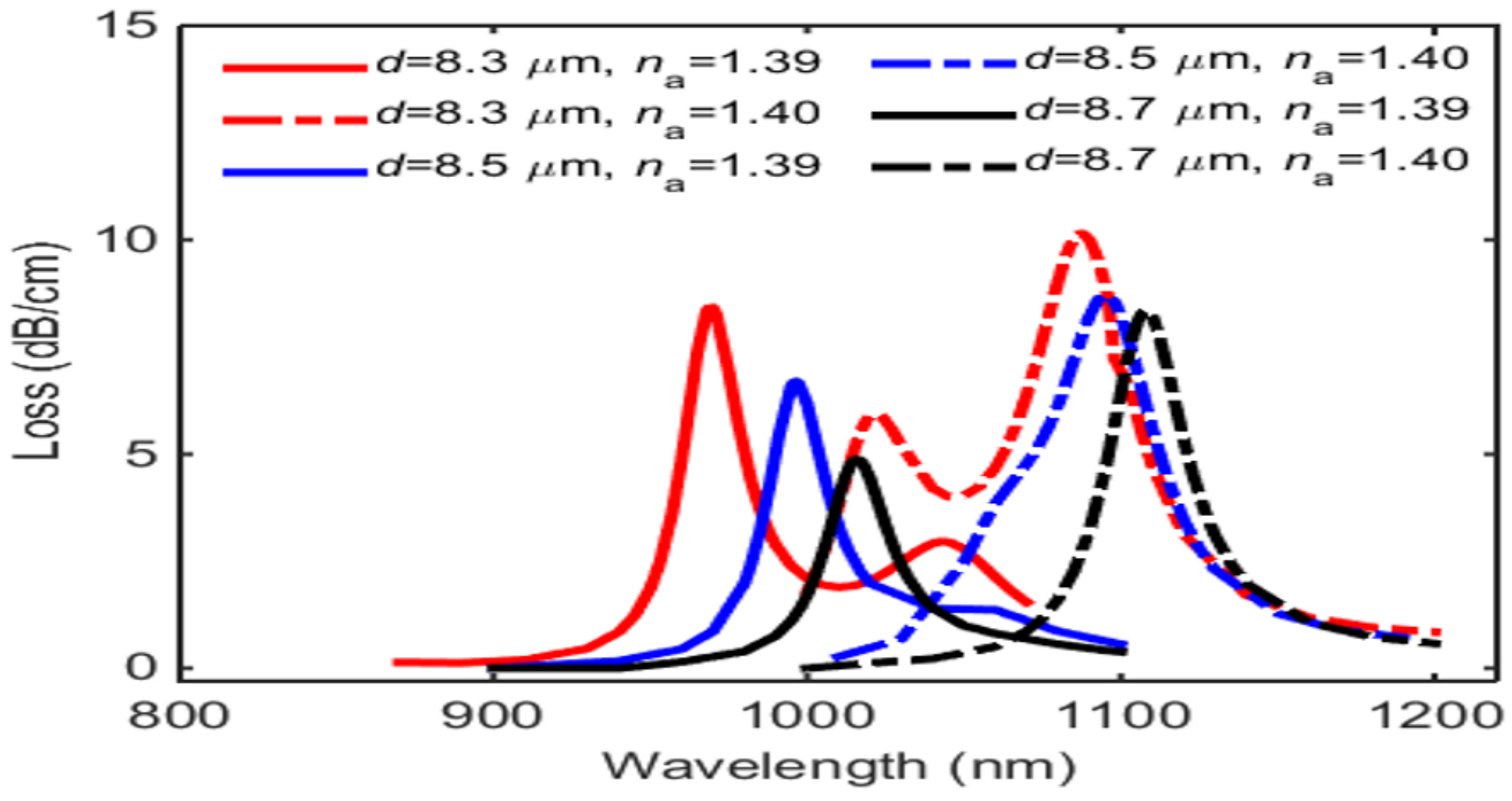

Figure 7

Loss spectrum of the core mode for different $d$ 

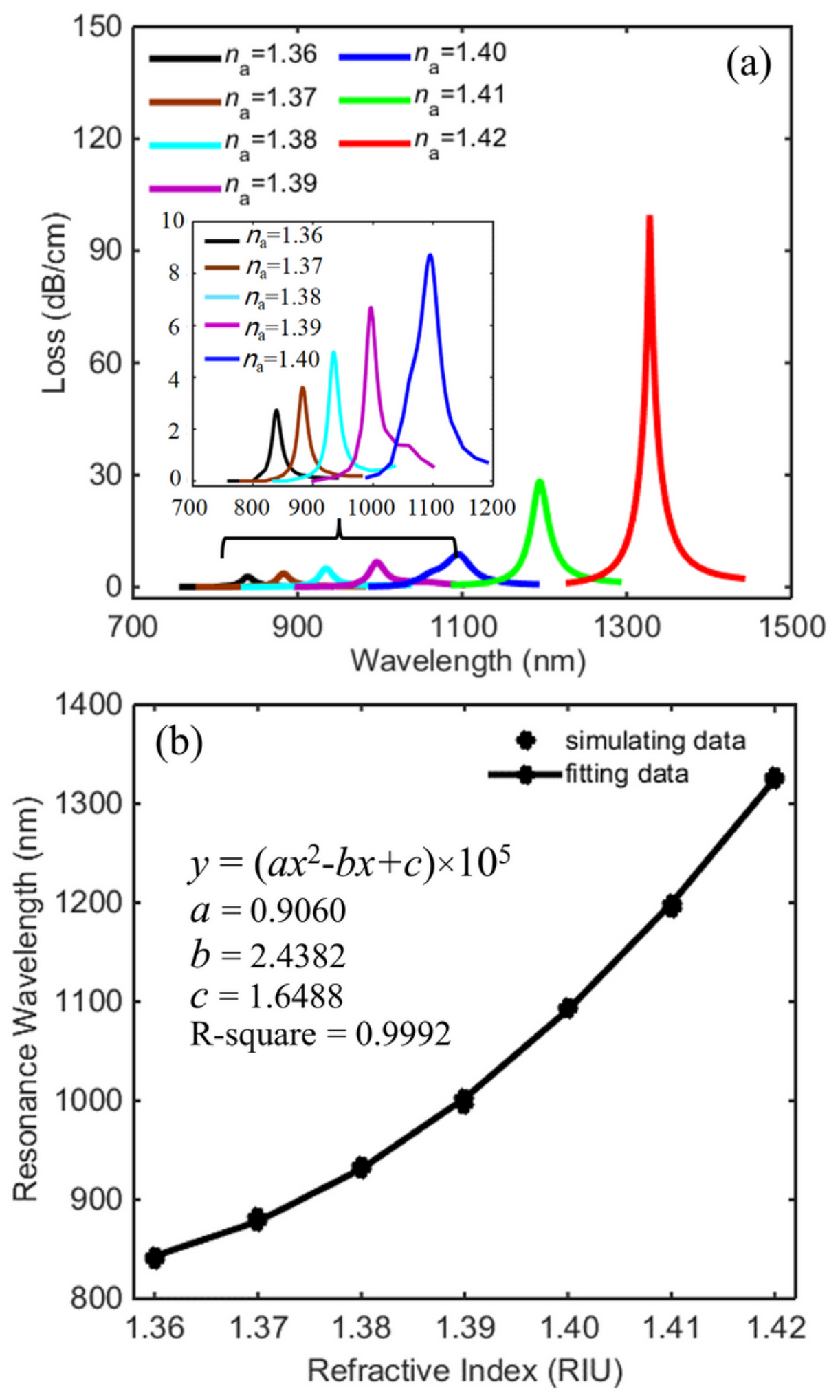

Figure 8

(a) Loss spectrum of the core mode for different na (b) Fitting curve of the resonance wavelength in relation to na 